Pacific Journal of Mathematic 


\title{
UNBOUNDED INVERSES OF HYPONORMAL OPERATORS
}

\author{
C. R. Putnam
}

\begin{abstract}
It is shown that certain unbounded inverses of hyponormal operators have Cartesian representations in which the real part is absolutely continuous and the imaginary part is bounded. An example is given which shows that in general the imaginary part is not absolutely continuous.
\end{abstract}

A bounded operator $T$ on a Hilbert space $\mathfrak{S C}_{\text {is }}$ said to be hyponormal if

$$
T^{*} T-T T^{*} \geqq 0
$$

For properties of such operators, see Putnam [2]. Such an operator is said to be completely hyponormal if there exists no nontrivial subspace of $\mathfrak{S}$ which reduces $T$ and on which $T$ is normal. Recall that a self-adjoint operator $A$ with the spectral resolution $A=\int \lambda d E_{i}$ is said to be absolutely continuous if $\left\|E_{\lambda} x\right\|^{2}$ is an absolutely continuous function of $\lambda$ for all $x$ in $\mathfrak{S}$. If $T$ is completely hyponormal with the Cartesian representation

$$
T=H+i J
$$

then both $H$ and $J$ are absolutely continuous; see [2, p. 42].

In case 0 is not in the spectrum of $T$ then $T^{-1}$ is also hyponormal; Stampfli [7]. Further,

$$
\left\|T^{-1}\right\|=d^{-1} \text { and }\|T x\| \geqq d\|x\|, x \in \mathfrak{S} \text { and } d=\operatorname{dist}(0, \operatorname{sp}(T)) .
$$

Suppose however that 0 is in the continuous spectrum of $T$, so that $T^{-1}$ exists as an unbounded operator, is closed, and $\mathfrak{D}_{T-1}=\mathfrak{R}_{T}$ is dense in $\mathfrak{S}$; cf. Stone [9, pp. 40, 129]. Then it was shown by Stampfli [8] that

$$
\mathfrak{D}_{T^{-1}} \subset \mathfrak{D}_{T^{-1 *}} \text { and }\left\|T^{-1^{*}} x\right\| \leqq\left\|T^{-1} x\right\| \text { for } x \in \mathfrak{D}_{T-1} .
$$

Thus $T^{-1}$ still behaves to a certain extent as does $T$. The question arises however as to whether $T^{-1}$ admits a Cartesian representation $T^{-1}=K+i L$, where $K$ and $L$ are self-adjoint, and also, if such a representation exists, whether these operators are absolutely continuous when $T$ is completely hyponormal. A partial answer is contained in the theorem below. 
It may be noted that if 0 belongs to the spectrum of $T$ and if $T$ is completely hyponormal, then 0 cannot be in the point spectrum of $T$, so that $T^{-1}$ exists. To see this, note that if $T x=0, x \neq 0$, then (1.1) implies that $T^{*} x=0$, and so the vector $x$ determines a normal reducing subspace of $T$.

THEOREM. Let $T$ be a (bounded) completely hyponormal operator and suppose that $0 \in \mathrm{sp}(T)$. In addition, suppose that there exists a number $\alpha>0$ for which the two open disks $|z \pm i \alpha|<\alpha$ contain no points of $\mathrm{sp}(T)$. Then 0 is in the continuous spectrum of $T$, and $T^{-1}$ has the representation

$$
T^{-1}=K+i L, K \text { and } L \text { self-adjoint and } L \text { bounded . }
$$

In particular, $T^{-1^{*}}=K-i L$ and (cf. (1.4))

$$
\left\|T^{-1^{*}} x\right\| \leqq\left\|T^{-1} x\right\|, x \in \mathfrak{D}_{T^{-1}}\left(=\mathfrak{D}_{T^{-1 *}}=\mathfrak{D}_{K}\right) .
$$

Further,

$K$ is absolutely continuous,

but

$$
L \text { need not be absolutely continuous . }
$$

REMARKs. The hypothesis of the theorem assures that 0 is in the spectrum of $T$ but that a small neighborhood of 0 does not contain too much spectrum. In view of the complete hyponormality assumption, however, any neighborhood of 0 necessarily intersects $\mathrm{sp}(T)$ in a set of positive measure; Putnam [5].

2. Proof of (1.5). It was noted above that 0 cannot be in the point spectrum of $T$. In view of the hypothesis concerning the disks it also follows that 0 is not in the residual spectrum of $T$. For, otherwise, $\mathfrak{D}_{T-1}=\Re_{T}$ is not dense and $T^{*} x=0$ for some $x \neq 0$. But, if $z \neq 0$ and if $z$ is real and sufficiently small, then $\left\|\left(T^{*}-i z I\right)^{-1}\right\|=$ $1 /$ dist $\left(i z, \operatorname{sp}\left(T^{*}\right)\right)=|z|^{-1}$, and this implies that $T x=0$, so that $x$ determines a normal reducing subspace of $T$, a contradiction; see Putnam [3], Stampfli [8], Sz.-Nagy and C. Foias [11].

Thus, 0 is in the continuous spectrum of $T, T^{-1}$ is closed, and $\mathfrak{D}_{T-1} \subset \mathfrak{D}_{T-1^{*}}($ cf. $(1.4))$. Clearly,

$$
\begin{aligned}
T^{-1} & =K+i L_{1}, \text { where } K=\frac{1}{2}\left(T^{-1}+T^{-1^{*}}\right) \text { and } \\
L_{1} & =(1 / 2 i)\left(T^{-1}-T^{-1^{*}}\right) .
\end{aligned}
$$

Note that 


$$
\mathfrak{D}_{K}=\mathfrak{D}_{L_{1}}=\mathfrak{D}_{T-1} \cap \mathfrak{D}_{T-1^{*}}=\mathfrak{D}_{T-1} .
$$

Moreover, $K^{*}=\frac{1}{2}\left(T^{-1}+T^{-1^{*}}\right)^{*} \supset \frac{1}{2}\left(T^{-1^{*}}+T^{-1^{* *}}\right)=\frac{1}{2}\left(T^{-1^{*}}+T^{-1}\right)=K$, and, similarly, $L_{1}^{*} \supset L_{1}$ (see, e,g., Sz.-Nagy [10, p. 29], so that both $K$ and $L_{1}$ are symmetric.

Suppose that $z \notin \mathrm{sp}(T)$ (and hence $z \neq 0$ ). Then $z(z I-T)^{-1} T=$ $\left(I-z^{-1} T\right)^{-1} T=\left[T^{-1}\left(I-z^{-1} T\right)\right]^{-1}=\left[T^{-1}-z^{-1} I\right]^{-1}$ and hence $z^{-1} \notin \operatorname{sp}\left(T^{-1}\right)$. (Concerning the spectrum of an unbounded operator, see Stone [9, p. 129]; Taylor [12, pp. 199-200]. Further, if $z \neq 0$ and $z^{-1} \notin \mathrm{sp}\left(T^{-1}\right)$, then $(z I-T)^{-1}=z^{-1} I-z^{-2}\left(z^{-1} I-T^{-1}\right)^{-1}$, so that for $z \neq 0, z \in \operatorname{sp}(T)$ if and only if $z^{-1} \in \operatorname{sp}\left(T^{-1}\right)$. (Cf. Taylor [12], loc. cit.) Since the mapping $w=1 / z$ sends the circles $|z+i \alpha|<\alpha$ and $|z-i \alpha|<\alpha(\alpha>0)$ respectively onto half-planes $\operatorname{Im}(w)>\beta$ and $\operatorname{Im}(w)<-\beta$ for some $\beta=$ const. $>0$, it follows that $\mathrm{sp}\left(T^{-1}\right)$ lies between the two lines $\operatorname{Im}(w)= \pm \beta$.

Next, it will be shown that if $z \notin \mathrm{sp}\left(T^{-1}\right)$ (hence $z^{-1} \notin \mathrm{sp}(T)$ ), then

$$
\left\|\left(T^{-1}-z I\right) x\right\| \geqq \operatorname{dist}\left(z, \operatorname{sp}\left(T^{-1}\right)\right)\|x\| \text { for } x \in \mathfrak{D}_{T-1} \text {. }
$$

To see this, note that $\left(T^{-1}-z I\right)^{-1}=\left[T^{-1}(I-z T)\right]^{-1}=(I-z T)^{-1} T=$ $z^{-1}\left[(I-z T)^{-1}-I\right]$, so that $\left(T^{-1}-z I\right)^{-1}$ is hyponormal. Also, by (1.3), $\left\|\left(T^{-1}-z I\right)^{-1}\right\|=1 / \operatorname{dist}\left(z, \mathrm{sp}\left(T^{-1}\right)\right)$, and relation (2.3) then follows. See also Clancey [1].

Let $W_{T^{-1}}$ denote the closure of the set $\left\{\left(T^{-1} x, x\right) ; x \in D_{T^{-1}}\right.$ and $\left.\|x\|=1\right\}$. It will be shown that $W_{T^{-1}}$ is contained in the least closed convex set containing $\mathrm{sp}\left(T^{-1}\right)$. It is sufficient to show that if $\operatorname{Re}\left(\operatorname{sp}\left(T^{-1}\right)\right) \leqq 0$ and if $a+i b \in W_{T^{-1}}$ then $a \leqq 0$. (Note that for this argument one can replace $T^{-1}$ by $r T^{-1}+s I$, where $r$ and $s$ are constants.) But if this is not the case then there exists some $x \in \mathfrak{D}_{T^{-1}},\|x\|=1$, such that $T^{-1} x=(a+i b) x+y$ with $(x, y)=0$ and $a>0$. Then for $c>0$, it follows from (2.3) that $c^{2} \leqq\left\|\left(T^{-1}-c I\right) x\right\|^{2}=(a-c)^{2}+b^{2}+\|y\|^{2}$ and hence $2 a c \leqq a^{2}+b^{2}+\|y\|^{2}$, which is impossible for large $c$. (This argument was used by Stampfli [7] for bounded hyponormal operators.)

It was noted earlier that $\mathrm{sp}\left(T^{-1}\right)$ lies in some strip $-\beta \leqq$ $\operatorname{Im}(w) \leqq \beta, \beta=$ const. $>0$, and it row follows that the set $W_{T^{-1}}$ does also. It follows from $(2.1)$ that $-\beta \leqq\left(L_{1} x, x\right) \leqq \beta$ for $\|x\|=1, x \in \mathfrak{D}_{L_{1}}\left(=\mathfrak{D}_{T^{-1}}=\right.$ $\Re_{T}$ ), so that $L_{1}$ is bounded on its (dense) domain. Consequently,

$$
L_{1} \text { has a unique bounded self-adjoint extension } L \text {. }
$$

Next, it will be shown that $K$ of (2.1) is self-adjoint. To this end, it is sufficient to show that

$$
\Re_{K+i k I}=\mathfrak{S} \text { holds for } k= \pm j \text { and some } j>0 \text {. }
$$

(See, e.g., Sz.-Nagy [10, pp. 37-38].) Since $\mathfrak{D}_{T^{-1}}=\mathfrak{D}_{K}=\mathfrak{D}_{L_{1}}$, then 
$K+i k I=T^{-1}+i\left(k I-L_{1}\right)=T^{-1}+i(k I-L)$ and so

$$
K+i k I=[I+i(k I-L) T] T^{-1} \text {. }
$$

(Note that all equations are interpreted in the strict operator sense.) Since $\mathfrak{R}_{T-1}=\mathfrak{D}_{T}=\mathfrak{S}$, then, in order to prove (2.5), it is sufficient to show that the (bounded) operator $I+i(k I-L) T$ is nonsingular for $|k|$ sufficiently large.

Choose $k$ so that

$$
|k|>\|L\|,
$$

and hence $(k I-L)$ is nonsingular. It is then sufficient to show that $Q=T-i(k I-L)^{-1}$ is nonsingular, that is,

$$
\|Q x\| \geqq c\|x\|,\left\|Q^{*} x\right\| \geqq c\|x\| \text { for some } c=\text { const. }>0,
$$

whenever $k$ is sufficiently large.

Next, choose $m$ real and satisfying $0<|m|<\alpha / 2$, where $\alpha$ is defined in the statement of the theorem. Then $\|(T-i m I) x\| \geqq|m|$ for $\|x\|=1$, in view of the present hypothesis and (1.3). Since $Q=$ $(T-i m I)-i\left[(k I-L)^{-1}-m I\right]$, it follows that, for $\|x\|=1,\|Q x\| \geqq$ $|m|-\left\|(k I-L)^{-1}-m I\right\|$. Since $(k I-L)^{-1}$ is definite, it is clear that by choosing $m$ to have the same sign as $k$, one has $\left\|(k I-L)^{-1}-m I\right\|<$ $|m|$, provided $k$ is sufficiently large, and hence the first relation of (2.8) is satisfied. Since $Q^{*}=\left(T^{*}+i m I\right)+i\left[(k I-L)^{-1}-m I\right]$, the second relation is clear from a similar argument if one notes that $\left\|(T-i m I)^{-1}\right\|=\left\|\left(T^{*}+i m I\right)^{-1}\right\|$ and hence $\left\|\left(T^{*}+i m I\right) x\right\| \geqq|m|$ for $\|x\|=1,0<|m|<\alpha / 2$. This completes the proof of (1.5).

3. Proof of (1.7). Let $K$ have the spectral resolution

$$
K=\int \lambda d G_{\lambda} \text {. }
$$

For any finite interval $\Delta$ and for any operator $A$ (possibly unbounded), let $A_{\Delta}=G(\Delta) A G(\Delta)$, as an operator on $G(\Delta) \mathcal{S}$. In order to prove that $K$ is absolutely continuous (on $\mathfrak{F}$ ) it is clearly sufficient to show that the (bounded) operator $K_{\Delta}$ is absolutely continuous on $G(\Delta) \mathfrak{S}_{\mathcal{C}}$ for every finite interval $\Delta$.

In order to show this, it is sufficient to show that the bounded operator $S(\Delta) \equiv G(\Delta) T^{-1} G(\Delta)=K_{\Delta}+i L_{\Delta}$ is completely hyponormal (cf. $\S 1$ above). If this is not the case however, then there exists a subspace $\mathfrak{S}_{1} \subset G(\Delta) \mathfrak{F}_{2}, \mathfrak{F}_{1} \neq 0$, with the property that $\mathfrak{F}_{1}$ reduces $S(\Delta)$ and $S(\Delta) / \mathfrak{S}_{1}$ is normal. It will be shown that this implies that

$$
\mathfrak{F}_{1} \text { reduces } T \text { and } T / \mathscr{S}_{1} \text { is normal, }
$$


thus contradicting the hypothesis that $T$ is completely hyponormal. To this end, note that in view of (1.5) and (1.6),

$$
\left\|T^{-1} x\right\|^{2}-\left\|T^{-1^{*}} x\right\|^{2}=2 i[(L x, K x)-(K x, L x)] \geqq 0, x \in \mathfrak{D}_{K} .
$$

Since, for every finite interval $\Delta, G(\Delta) x \in \mathfrak{D}_{K}\left(x \in \mathfrak{S}_{2}\right)$, then (3.3) implies that

$$
i\left(K_{\Delta} L_{\Delta}-L_{\Delta} K_{4}\right) \equiv M^{(\Delta)} \geqq 0,
$$

where now all operators of (3.4) are bounded. It is clear that $M^{(\Delta)}=$ $G(\Delta) M^{(\Delta)} G(\Delta)$ and that if $\eta$ is any interval containing $\Delta$ then $G(\Delta) M^{(\eta)} G(\Delta)=M^{(\Delta)} \geqq 0$. Since $M^{(\Delta)} x=0$ for $x \in \mathfrak{K}_{1}$, then $M^{(\eta)} G(\Delta) \mathfrak{K}_{1}=0$, that is, $M^{(\eta)} \mathfrak{K}_{1}=0$. Since $\mathfrak{F}_{1}$ reduces $S(\Delta)$, and hence also $K_{\Delta}$, then $\mathfrak{S}_{1}$ is invariant under $K_{\eta}$ (note that $K_{\eta} \mathfrak{S}_{1}=K_{4} \mathfrak{S}_{1} \subset \mathfrak{S}_{1}$ ), thus $M^{(\eta)} K_{\eta} \mathfrak{S}_{1}=0$. But (3.4) (with $\triangle$ replaced by $\eta$ ) implies that $i\left(K_{\eta}^{2} L_{\eta}-L_{\eta} K_{\eta}^{2}\right)=$ $\left(K_{\eta} M^{(\eta)}+M^{(\eta)} K_{\eta}\right)$, so that $K_{\eta}^{2} L_{\eta} x=L_{\eta} K_{\eta}^{2} x$ for $x$ in $\mathfrak{S}_{1}$. In the same way, one obtains $K_{\eta}^{n} L_{\eta} x=L_{\eta} K_{\eta}^{n} x(n=0,1,2, \cdots)$ for $x$ in $\mathfrak{F}_{1}$. Since $G(\Delta)$ is the strong limit of polynomials in $K_{\eta}$ (note that $\eta$ contains $\Delta$ ), this implies that $G(\Delta) L x=G(\eta) L x$ for $x$ in $\mathscr{S}_{1}$. Since $\eta$ is any interval containing $\Delta$, it follows that $G(\Delta) L \mathfrak{S}_{1}=L \mathscr{S}_{1}$. But $\mathfrak{S}_{1}$ reduces $S(\Delta)$, hence also $G(\Delta) L G(\Delta)$, so that $G(\Delta) L \mathfrak{F}_{1}=G(\Delta) L G(\Delta) \mathscr{F}_{1} \subset \mathfrak{F}_{1}$. Thus $\mathfrak{F}_{1}$ reduces $L$ (as well as $K$, since $K \mathfrak{S}_{1}=K_{\Delta} \mathfrak{F}_{1} \subset \mathfrak{S}_{1}$ ). Thus $\mathfrak{S}_{1}$ reduces $T^{-1}$. Also, since $M^{(4)} \mathfrak{K}_{1}=0$, it follows from (3.3) and (3.4) that $\left\|T^{-1} x\right\|=\left\|T^{-1^{*}} x\right\|$ for $x$ in $\mathfrak{S}_{1}(\subset G(\Delta) \mathfrak{S})$. Thus $T^{-1}$ is normal on $\mathfrak{S}_{1}$ (cf. Sz.-Nagy [10, p. 33]) and hence (3.2) follows. As noted earlier, this yields a contradiction, and, as also noted before, relation (1.7) follows.

4. Proof of (1.8). Let $Q$ denote the Hilbert transform on $\mathfrak{S}=$ $L^{2}(-\infty, \infty)$ defined by

$$
(Q x)(t)=(i \pi)^{-1} \int_{-\infty}^{\infty}(s-t)^{-1} x(s) d s,
$$

the integral being a Cauchy principal value. It is well-known that $Q$ is both $\mathbf{u}$ itary and self-adjoint and that its spectrum consists of \pm 1 , each of infinite multiplicity. Define the self-adjoint operators $K$ and $L$ on $L^{2}(-\infty, \infty)$ by

$$
K=t, L=-2 I-Q .
$$

The spectrum of the multiplication operator $K$ is $(-\infty, \infty)$, while that of the (bounded) operator $L$ consists of the two numbers -1 and -3 , each of infinite multiplicity. Further, $K$ is absolutely continuous, but $L$ is not. Next, define $S$ by

$$
S=K+i L \text {. }
$$


Since $L$ is bounded, $S^{*}=K-i L$ and $\mathfrak{D}_{S}=\mathfrak{D}_{S^{*}}=\mathfrak{D}_{K}$. It will be shown that $T=S^{-1}$ exists, is bounded and is hyponormal.

First, note that 0 is not in the point spectrum of either $S$ or $S^{*}$. For, if $x \in \mathfrak{D}_{S}$ and if $S x=0$, then $0=(S x, x)=(K x, x)+i(L x, x)$ and hence $(L x, x)=0$. But this is impossible since $-3 I \leqq L \leqq-I$. Similarly, 0 is not in the point spectrum of $S^{*}$.

It follows that $T=S^{-1}$ exists and that $\mathfrak{D}_{T}=\mathfrak{D}_{S-1}=\Re_{S}$ is dense. Further, if $x \in \mathfrak{D}_{S}$, then $(S x, x)=(K x, x)+i(L x, x)$ and so $\|x\|^{2} \leqq$ $|(L x, x)| \leqq|(S x, x)| \leqq\|S x\|\|x\|$, that is, $\|S x\| \geqq\|x\|$. If $y=S x$, this implies that $\|T y\| \leqq\|y\|$ for $y \in D_{T}$, so that $T$ is bounded on its domain. Since $K$ is closed and $L$ is bounded, then $S$ is closed and hence (cf. Stone [9, p. 40]), $S^{-1}=T$ is closed. It follows that $T$ must be bounded (with $\mathfrak{D}_{T}=\mathfrak{F}=L^{2}(-\infty, \infty)$ ).

Next, it will be shown that

$$
\|S x\| \geqq\left\|S^{*} x\right\| \text { for } x \in \mathscr{D}_{S} .
$$

To see this, note that (cf. (3.3))

$$
\|S x\|^{2}-\left\|S^{*} x\right\|^{2}=2 i[(L x, K x)-(K x, L x)], x \in \mathfrak{D}_{S} .
$$

For any finite interval $\Delta$, let $x=G(\Delta) x$, where $K=t=\int \lambda d G_{\lambda}$. Then one obtains $\|S x\|^{2}-\left\|S^{*} x\right\|^{2}=2\left(M^{(\Delta)} x, x\right)$, where (cf. (3.4)) $M^{(\Delta)}=$ $i\left(K_{\Delta} L_{\Delta}-K_{\Delta} L_{\Delta}\right)=i\left(Q_{\Delta} K_{\Delta}-K_{\Delta} Q_{\Delta}\right)$ (cf. (4.2)) and

$$
\left(M^{(\Delta)} x, x\right)=\pi^{-1}\left|\int_{4} x(s) d s\right|^{2} \geqq 0 .
$$

Thus,

$$
\|S G(\Delta) x\|^{2}-\left\|S^{*} G(\Delta) x\right\|^{2} \geqq 0 \text { for } x \in \mathfrak{S},
$$

where $\Delta$ is any finite interval. If $x \in \mathfrak{D}_{S}\left(=\mathfrak{D}_{S^{4}}\right)$ and $\Delta_{n}=(-n, n)$, $n=1,2, \cdots$, then $G\left(\Delta_{n}\right) x \rightarrow x$ as $n \rightarrow \infty$. Moreover, since $L$ is bounded, it is clear from (4.3) that $S G\left(\Delta_{n}\right) x \rightarrow S x$ and $S^{*} G\left(\Delta_{n}\right) x \rightarrow S^{*} x$. Relation (4.4) now follows from (4.6).

By an argument similar to that used by Stampfli [8] (cf. (1.4) above), it follows that $T=S^{-1}$ is hyponormal; see Clancey [1, p. 33]. Since $-3 I \leqq L \leqq-I$, it follows that $\mathrm{sp}(S)$ lies between the lines $\operatorname{Im}(w)=-3$ and $\operatorname{Im}(w)=-1$ of the $w$-plane (cf. Taylor [12, p. 199]; Clancey [1, p. 34]) and hence $\mathrm{sp}(T)$ lies between the circles in the $z$-plane which are images of these lines under the mapping $z=1 / w$. (These circles are centered on the positive imaginary axis and have the real axis as a common tangent at $z=0$.) In case $T$ is completely hyponormal, it can clearly be identified with the operator occurring in the statement of the Theorem.

Suppose then that $T$ is not completely hyponormal. Then $T$ 
cannot be normal. For, otherwise (cf. (3.3)), $(L x, K x)=(K x, L x)$ for all $x \in L^{2}(-\infty, \infty)$, where $L, K$ are defined by (4.2). Thus, $(Q x, K x)=$ $(K x, Q x)$ for all $x \in L^{2}(-\infty, \infty)$ and, in particular, if $\Delta$ is any finite interval and if $x$ is replaced by $G(\Delta) x$, it follows that $K_{\Delta} Q_{\Delta}-Q_{\Delta} K_{\Delta}=0$, where $A_{\Delta}=G(\Delta) A G(\Delta)$. Thus, for any $x \in L^{2}(-\infty, \infty), \int_{\Delta} x(t) d t=0, \Delta$ arbitrary, which is clearly impossible.

Since $T$ is not normal, its normal part (if it exists, i.e., if $T$ is not completely hyponormal) can be split off, so that $T$ can be represented as

$$
T=T_{1} \oplus T_{2}
$$

where $T_{1}$ is normal and $T_{2}$ is completely hyponormal. Further, it is clear that $T^{-1}=T_{1}^{-1} \oplus T_{2}^{-1}$ and that $T_{2}$ has the properties of $T$ in the statement of the theorem. If $T_{2}^{-1}=K_{2}+i L_{2}$ as in the theorem, then, since $T^{-1}=K+i L=T_{1}^{-1} \oplus\left(K_{2}+i L_{2}\right)$, it follows that $L=$ $(1 / 2 i)\left(T_{1}^{-1}-T_{1}^{-1^{*}}\right) \oplus L_{2}$. In particular, $\mathrm{sp}\left(L_{2}\right)$ is a subset of $\mathrm{sp}(L)$, so that $\operatorname{sp}\left(L_{2}\right)$ contains at most the two numbers -1 and -3 , and hence $L_{2}$ is not absolutely continuous.

Thus, an operator $T$ satisfying the conditions of the theorem has been constructed for which $L$ of (1.5) is not absolutely continuous. This completes the proof of the theorem.

5. Remarks. In case $T$ is a (bounded) hyponormal operator and if the spectrum of its imaginary part has measure zero then $T$ must be normal; see [2, p. 43]. That the corresponding assertion can be false if $T$ is unbounded, even if $T$ admits the representation (1.5) (with $T^{-1}$ replaced there by $T$ ), is clear from the example constructed above. Also, if $T$ is (bounded and) hyponormal, the spectra of its real and imaginary parts are precisely the projections onto the coordinate axes of the spectrum of $T$; [2, p. 46]. This is not in general true in the unbounded case. (Concerning the connection between the spectrum of $T$ and that of its real or imaginary part when $T$ is unbounded and is, in some sense, "hyponormal," see Clancey [1].) Roughly speaking, if $T$ has a representation $T=K+i L$, where $K$ and $L$ are self-adjoint, then, even if $L$ is bounded, its spectrum is largely unpredictable unless $\mathrm{sp}(K) \neq(-\infty, \infty)$. (In this connection, see Clancey [1, Th. 4.2.6]; Putnam [2, p. 39], [4]; Rosenblum [6].) Thus, it is not completely accidental that the operator $S$ of $\S 4$ above has a real part with spectrum equal to the entire real axis.

\section{REFERENCES}

1. K.F. Clancey, Spectral properties of semi-normal operators, Thesis, Purdue University, January, 1969. 
2. C. R. Putnam, Commutation properties of Hilbert space operators and related topics, Ergebnisse der Math. 36 (1967), Springer.

3. — Eigenvalues and boundary spectra, Illinois J. Math. 12 (1968), 278-282.

4. —, Absolute continuity of singular integral operators, Amer. J. Math. 91 (1969), 453-462.

5. - An inequality for the area of hyponormal spectra, Math. Zeits. 116 (1970), 323-330.

6. M. Rosenblum, A spectral theory for self-adjoint singular integral operators, Amer. J. Math. 86 (1966), 314-328.

7. J.G. Stampfli, Hyponormal operators and spectral density, Trans. Amer. Math. Soc. 117 (1965), 469-476.

8. - Analytic extensions and spectral localization, J. Math. Mech. 16 (1966), $287-296$.

9. M. H. Stone, Linear transformations in Hilbert space and their application to analysis, Amer. Math. Soc., New York, 1932.

10. B. Sz.-Nagy, Spectraldarstellung linearer Transformationen des Hilbertschen Raumes, Ergebnisse der Math. 39 (1967), Springer.

11. B. Sz.-Nagy and C. Foias, Une relation parmi les vecteurs propres d'un operateur de l'espace de Hilbert et de l'operateur adjoint, Acta Szeged 20 (1959), 91-96.

12. A. E. Taylor, Spectral theory of closed distributive operators, Acta Math. 84 (1951), 189-224.

Received May 11, 1970. This work was supported by a National Science Foundation research grant.

Purdue University 


\section{PACIFIC JOURNAL OF MATHEMATICS}

\section{EDITORS}

\author{
H. SAMELSON \\ Stanford University \\ Stanford, California 94305 \\ Richard Pierce \\ University of Washington \\ Seattle, Washington 98105
}

\author{
J. DugundJI \\ Department of Mathematics \\ University of Southern California \\ Los Angeles, California 90007 \\ RICHARD ARENS \\ University of California \\ Los Angeles, California 9.0024
}

\section{ASSOCIATE EDITORS}

\begin{tabular}{|c|c|}
\hline E. F. BECKENBACH & K. YoshidA \\
\hline \multicolumn{2}{|c|}{ SUPPORTING INSTITUTIONS } \\
\hline UNIVERSITY OF BRITISH COLUMBIA & STANFORD UNIVERSITY \\
\hline CALIFORNIA INSTITUTE OF TECHNOLOGY & UNIVERSITY OF TOKYO \\
\hline UNIVERSITY OF CALIFORNIA & UNIVERSITY OF UTAH \\
\hline MONTANA STATE UNIVERSITY & WASHINGTON STATE UNIVERSITY \\
\hline UNIVERSITY OF NEVADA & UNIVERSITY OF WASHINGTON \\
\hline NEW MEXICO STATE UNIVERSITY & $* \quad * \quad *$ \\
\hline OREGON STATE UNIVERSITY & AMERICAN MATHEMATICAL SOCIETY \\
\hline UNIVERSITY OF OREGON & CHEVRON RESEARCH CORPORATION \\
\hline OSAKA UNIVERSITY & TRW SYSTEMS \\
\hline UNIVERSITY OF SOUTHERN CALIFORNIA & NAVAL WEAPONS CENTER \\
\hline
\end{tabular}

The Supporting Institutions listed above contribute to the cost of publication of this Journal, but they are not owners or publishers and have no responsibility for its content or policies.

Mathematical papers intended for publication in the Pacific Journal of Mathematics should be in typed form or offset-reproduced, (not dittoed), double spaced with large margins. Underline Greek letters in red, German in green, and script in blue. The first paragraph or two must be capable of being used separately as a synopsis of the entire paper. The editorial "we" must not be used in the synopsis, and items of the bibliography should not be cited there unless absolutely necessary, in which case they must be identified by author and Journal, rather than by item number. Manuscripts, in duplicate if possible, may be sent to any one of the four editors. Please classify according to the scheme of Math. Rev. Index to Vol. 39. All other communications to the editors should be addressed to the managing editor, Richard Arens, University of California, Los Angeles, California, 90024.

50 reprints are provided free for each article; additional copies may be obtained at cost in multiples of 50 .

The Pacific Journal of Mathematics is published monthly. Effective with Volume 16 the price per volume (3 numbers) is $\$ 8.00$; single issues, $\$ 3.00$. Special price for current issues to individual faculty members of supporting institutions and to individual members of the American Mathematical Society: $\$ 4.00$ per volume; single issues $\$ 1.50$. Back numbers are available.

Subscriptions, orders for back numbers, and changes of address should be sent to Pacific Journal of Mathematics, 103 Highland Boulevard, Berkeley, California, 94708.

PUBLISHED BY PACIFIC JOURNAL OF MATHEMATICS, A NON-PROFIT CORPORATION

Printed at Kokusai Bunken Insatsusha (Internatıonal Academic Printing Co., Ltd.), 7-17, Fujimi 2-chome, Chiyoda-ku, Tokyo, Japan. 


\section{Pacific Journal of Mathematics}

\section{Vol. 35, No. $3 \quad$ November, 1970}

John D. Arrison and Michael Rich, On nearly commutative degree one algebras . . . 533

Bruce Alan Barnes, Algebras with minimal left ideals which are Hilbert spaces . . . . 537

Robert F. Brown, An elementary proof of the uniqueness of the fixed point index . . . 549

Ronn L. Carpenter, Principal ideals in F-algebras .................... 559

Chen Chung Chang and Yiannis (John) Nicolas Moschovakis, The Suslin-Kleene

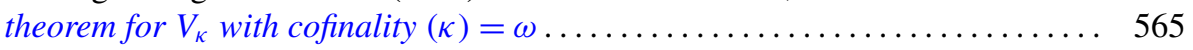

Theodore Seio Chihara, The derived set of the spectrum of a distribution

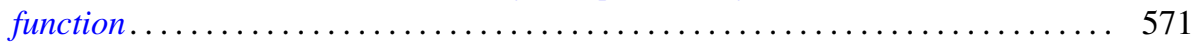

Tae Geun Cho, On the Choquet boundary for a nonclosed subspace of $C(S) \ldots \ldots \quad 575$

Richard Brian Darst, The Lebesgue decomposition, Radon-Nikodym derivative,

conditional expectation, and martingale convergence for lattices of sets .......

David E. Fields, Dimension theory in power series rings . . . . . . . . . . . .

Michael Lawrence Fredman, Congruence formulas obtained by counting

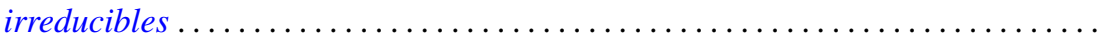

John Eric Gilbert, On the ideal structure of some algebras of analytic functions.....

G. Goss and Giovanni Viglino, Some topological properties weaker than

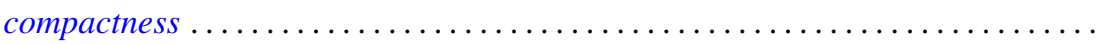

581

601

625

George Grätzer and J. Sichler, On the endomorphism semigroup (and category) of

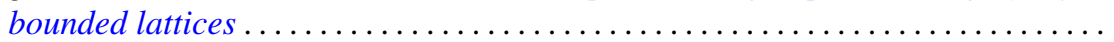

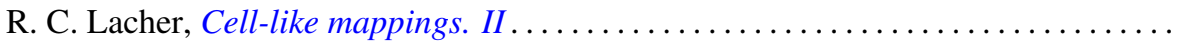

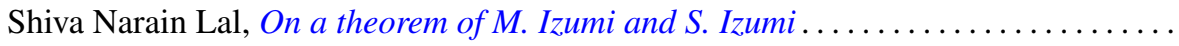

661

Howard Barrow Lambert, Differential mappings on a vector space ...............

Richard G. Levin and Takayuki Tamura, Notes on commutative power joined

semigroups.

Robert Edward Lewand and Kevin Mor McCrimmon, Macdonald's theorem for quadratic Jordan algebras.

J. A. Marti, On some types of completeness in topological vector spaces ....

Walter J. Meyer, Characterization of the Steiner point

717

Saad H. Mohamed, Rings whose homomorphic images are $q$-rings ...

727

Thomas V. O'Brien and William Lawrence Reddy, Each compact orientable surface

of positive genus admits an expansive homeomorphism ...

737

Robert James Plemmons and M. T. West, On the semigroup of binary relations...

743

Calvin R. Putnam, Unbounded inverses of hyponormal operator . .

755

William T. Reid, Some remarks on special disconjugacy criteria for differential

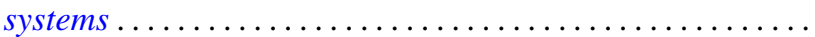

C. Ambrose Rogers, The convex generation of convex Borel sets in euclidean

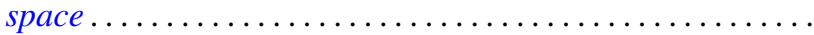

S. Saran, A general theorem for bilinear generating functions .

S. W. Smith, Cone relationships of biorthogonal systems ......

Wolmer Vasconcelos, On commutative endomorphism rings ....

795

Vernon Emil Zander, Products of finitely additive set functions from Orlicz

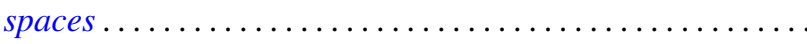

G. Sankaranarayanan and C. Suyambulingom, Correction to: "Some renewal

theorems concerning a sequence of correlated random variables" .

Joseph Zaks, Correction to: "Trivially extending decompositions of $E^{n}$ ”....... 805

Dong Hoon Lee, Correction to: "The adjoint group of Lie groups" ............ 805

James Edward Ward, Correction to: "Two-groups and Jordan algebras". 